\title{
Evolutionary Plasticity of AmrZ Regulation in Pseudomonas
}

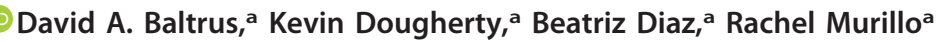 \\ aSchool of Plant Sciences, University of Arizona, Tucson, Arizona, USA
}

\begin{abstract}
Z encodes a master regulator protein conserved across pseudomonads, which can be either a positive or negative regulator of swimming motility depending on the species examined. To better understand plasticity in the regulatory function of AmrZ, we characterized the mode of regulation for this protein for two different motility-related phenotypes in Pseudomonas stutzeri. As in Pseudomonas syringae, AmrZ functions as a positive regulator of swimming motility within $P$. stutzeri, which suggests that the functions of this protein with regard to swimming motility have switched at least twice across pseudomonads. Shifts in mode of regulation cannot be explained by changes in AmrZ sequence alone. We further show that AmrZ acts as a positive regulator of colony spreading within this strain and that this regulation is at least partially independent of swimming motility. Closer investigation of mechanistic shifts in dual-function regulators like AmrZ could provide unique insights into how transcriptional pathways are rewired between closely related species.
\end{abstract}

IMPORTANCE Microbes often display finely tuned patterns of gene regulation across different environments, with major regulatory changes controlled by a small group of "master" regulators within each cell. AmrZ is a master regulator of gene expression across pseudomonads and can be either a positive or negative regulator for a variety of pathways depending on the strain and genomic context. Here, we demonstrate that the phenotypic outcomes of regulation of swimming motility by AmrZ have switched at least twice independently in pseudomonads, so that AmrZ promotes increased swimming motility in $P$. stutzeri and $P$. syringae but represses this phenotype in Pseudomonas fluorescens and Pseudomonas aeruginosa. Since examples of switches in regulatory mode are relatively rare, further investigation into the mechanisms underlying shifts in regulator function for AmrZ could provide unique insights into the evolution of bacterial regulatory proteins.

KEYWORDS AmrZ, motility, Pseudomonas stutzeri

rap ranscriptional regulation of bacterial operons is often tightly balanced to enable rapid phenotypic changes while minimizing energetic costs associated with overproduction of mRNA and proteins (1-3). Indeed, survival of bacterial populations requires gene regulatory schemes that can respond to fine-scale gradients and rapid shifts in environmental conditions, and data suggest that these regulatory schemes are optimized to appropriately respond to a variety of possible environments $(1,4,5)$. Dissection of the mechanisms of action and characterization of transcriptional responses for numerous repressors and activators across species has provided knowledge about the functions of these proteins while also creating a context for exploring how such pathways evolve (4-6). Although there exists great appreciation for the general plasticity of bacterial regulatory networks, our understanding of how particular pathways are transcriptionally rewired and how specific proteins change regulatory mode over evolutionary time remains far from complete. While much previous work has focused on defining how and when genes are regulated, there have been few examples that have pinpointed changes in the direction of regulation by the same protein across
Received 9 March 2018 Accepted 26 March 2018 Published 18 April 2018 Citation Baltrus DA, Dougherty K, Diaz B, Murillo R. 2018. Evolutionary plasticity of AmrZ regulation in Pseudomonas. mSphere 3:e0013218. https://doi.org/10.1128/mSphere.00132-18. Editor Craig D. Ellermeier, University of lowa Copyright $\odot 2018$ Baltrus et al. This is an openaccess article distributed under the terms of the Creative Commons Attribution 4.0 International license.

Address correspondence to David A. Baltrus, Baltrus@email.arizona.edu.

Multiple reversals of $A m r Z$ regulation of motility in Pseudomonas and a single amino acid change uncover independent mechanisms of positive regulation of phenotypes by AmrZ in Pseudomonas stutzeri. @surt_lab 
closely related lineages. Here, we further document an example in pseudomonads involving the ribbon-helix-helix transcriptional regulator AmrZ.

$a m r Z$, alginate and motility regulator $Z$, encodes a ribbon-helix-helix transcription factor that directly regulates a variety of pathways across Pseudomonas species and is a master regulator for numerous pathways associated with virulence in $P$. aeruginosa (7). Unlike the vast majority of characterized regulators, AmrZ can directly affect transcription both positively and negatively within the same cell, with the precise function depending on the intrinsic structure of the protein and the genomic context of the DNA binding site $(8,9)$. Moreover, although the mechanism of repression by AmrZ canonically involves binding to operator regions, how this protein specifically activates transcription remains unclear $(8,9)$. Regulatory pathways involving AmrZ are best described for Pseudomonas aeruginosa, where expression from amrZ is promoted by AlgT (10), activates 9 genes, and represses 49 genes, including itself (7). Regulation of AmrZ can also be affected posttranscriptionally by a small RNA, ersA (11). Pathways directly repressed by AmrZ include swimming motility and the operon controlling production of the exopolysaccharide Psl (7). Interestingly, AmrZ can differentially modulate construction of all three type VI secretion systems in P. aeruginosa (12). In contrast, AmrZ has been shown to promote expression of the alginate operon and type IV pilus-dependent twitching motility $(13,14)$. AmrZ has also been shown to influence colony morphology and biofilm formation, with these phenotypes mediated by AmrZ-dependent changes in cyclic di-GMP (7). In Pseudomonas fluorescens F113, amrZ mutants are hypermotile in swimming assays and iron uptake genes are derepressed (15), with many of these phenotypes appearing to be modulated by changes in cyclic di-GMP (16). In Pseudomonas syringae DC3000, AmrZ is a positive regulator of swimming motility as well as a variety of other virulence genes (17).

Pseudomonas stutzeri is an environmentally ubiquitous species known best as a denitrifier as well as for its diverse metabolic capabilities (18). Phylogenies of pseudomonads demonstrate that $P$. stutzeri is placed uniquely in between other species where impacts of AmrZ on swimming motility have been evaluated (see reference 19 and Fig. 4), and thus, information about the role of AmrZ from this species could polarize our understanding of regulatory modes for this protein. Given opposing information about the regulatory role of AmrZ across pseudomonads, the original aim of this paper was to test how AmrZ regulated swimming motility in P. stutzeri. We subsequently noticed that AmrZ also positively regulates a colony spreading phenotype in this species and that an allele of this gene enables the differentiation of positive regulation for these two phenotypes. Our results therefore provide a new viewpoint for understanding how regulatory pathways can evolve in bacteria.

\section{RESULTS AND DISCUSSION}

AmrZ is a well-studied regulator of phenotypes important for environmental survival across pseudomonads, including multiple virulence traits in $P$. aeruginosa $(7,15,17)$. Since $P$. stutzeri is uniquely phylogenetically positioned among the strains previously characterized for AmrZ-dependent regulation of motility, we investigated phenotypic effects of deleting $a m r Z$ in this bacterium. In both deletion lines (DBL1052 and DBL1053), loss of $a m r Z$ leads to decreased flagellar motility, clearly demonstrating that AmrZ is a positive regulator for this phenotype within this strain (Fig. 1A). However, that these lines display relatively higher motility than an immotile flgK deletion strain demonstrates that AmrZ is not the sole positive regulator for flagellar operons in P. stutzeri (Fig. 1B). Loss of motility can be complemented by expression of $a m r Z$ with its native promoter from the Tn7 site (Fig. 2A), and we note that complemented strains have levels of swimming motility at least equal to that of the wild type (https://figshare .com/s/2a79a1e48fa851d51577). This result directly contrasts with what has been reported across multiple other Pseudomonas species $(10,15)$ but supports the role of AmrZ as a positive regulator of motility in $P$. syringae (17). Using these same strain comparisons, we have also been able to show that AmrZ is a positive regulator of colony spreading in P. stutzeri (Fig. $1 \mathrm{C}$ and $\mathrm{D}$ and $2 \mathrm{~B}$ ). 


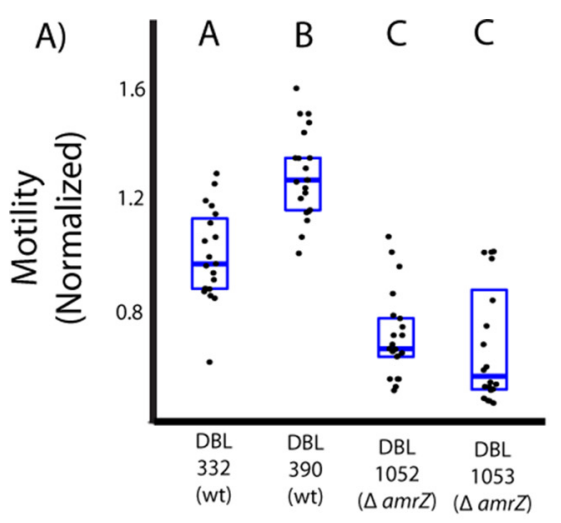

Strain
C)

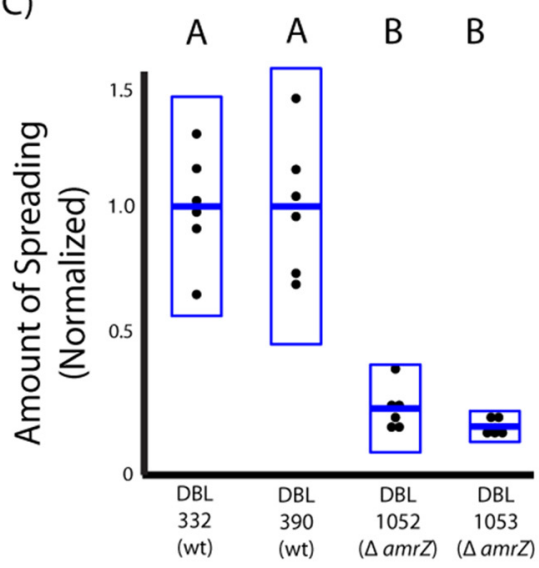

B)

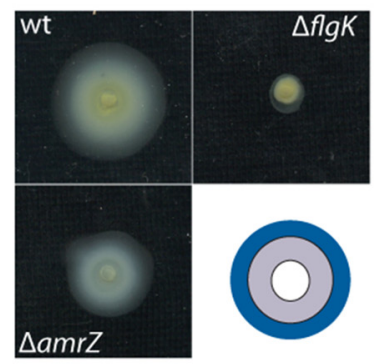

D)

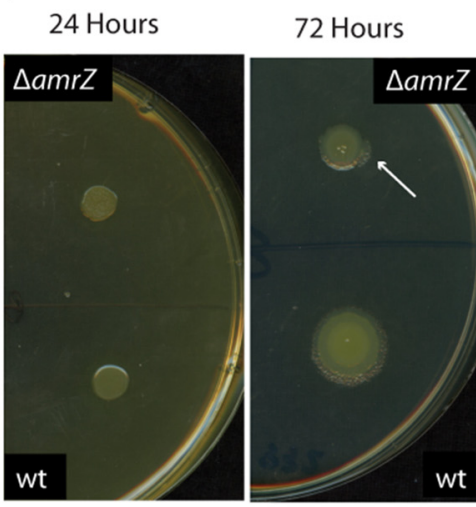

\section{Strain}

FIG $1 \mathrm{amrZ}$ is a positive regulator of swimming motility and colony spreading in Pseudomonas stutzeri. Strains DBL1052 and DBL1053 are derived from strains DBL332 and DBL390, respectively, and contain independently created deletions in $a m r Z$. Individual data points for each assay are plotted for each strain, with boxes representing 2 standard deviations and means plotted as horizontal blue lines at the center of the box plots. Measurements within each assay have been normalized so that the value of DBL332 is 1. Letters above each box plot indicate that mean values are significantly different at $P<0.01$ according to Tukey's HSD test. (A) AmrZ is a positive regulator of swimming motility, and loss of swimming motility is seen in independently created $a m r Z$ deletion lines. (B) Representative images from motility assays are shown for a wild-type strain, an $a m r Z$ deletion strain, and an flgK deletion strain. In each case, colored circles have been created that match halos in these pictures and have been superimposed in the final image (blue, DBL332; gray, DBL1052; white; DBL1168). (C) AmrZ is a positive regulator of colony spreading, and loss of spreading is seen in independently created $a m r Z$ deletion lines. (D) Representative example of colony spreading activity and positive regulation by AmrZ. The same plate is shown after being scanned after 1 day of growth (left) and after 3 days of growth (right). AmrZ ${ }^{+}$strains spread outward on $\mathrm{KB}$ medium over time, while $\mathrm{AmrZ}^{-}$strains fail to spread unless there are compensatory mutations (as shown by the white arrow in the DBL1052 strain). wt, wild type.

At this time, it is difficult to discern which molecular changes underlie shifts in AmrZ function in $P$. stutzeri because the mechanism of regulation by this protein for motility differs across pseudomonads. In $P$. aeruginosa, AmrZ binds upstream of fleQ in order to directly repress expression of fleQ; however, no AmrZ binding was observed upstream of fleQ in $P$. fluorescens $\mathrm{F} 113$ even though $a m r Z$ mutants are hypermotile $(7,15)$. As a first step to determine the molecular mechanism behind alterations in AmrZ-dependent regulation of motility, we tested whether the $P$. aeruginosa allele could complement the loss of motility in P. stutzeri. These alleles are slightly diverged from one another but overall maintain relatively high sequence similarity (see Fig. 4C), and our assays thus test whether a small number of protein structural changes are directly responsible for regulatory shifts in phenotypes. As shown in Fig. $2 A$ and $B$, the $P$. aeruginosa allele of $a m r Z$ is able to complement both the swimming motility and 
A)

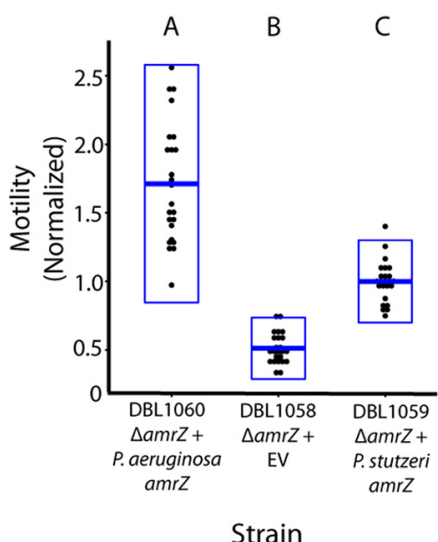

B)

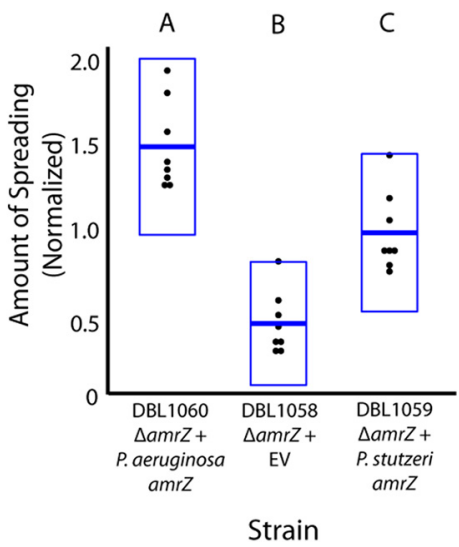

FIG 2 Alleles of $a m r Z$ from either $P$. stutzeri or $P$. aeruginosa can phenotypically complement an $a m r Z$ deletion strain in trans. Strains DBL1058, DBL1059, and DBL1060 are derived from strain DBL830 ( $\triangle a m r Z)$. DBL1058 contains an empty-vector gentamicin resistance cassette, while DBL1059 contains amr $Z$ (native promoter) integrated into the Tn7 site of the chromosome. DBL1060 contains the $a m r Z$ allele from $P$. aeruginosa (native promoter from $P$. stutzeri) integrated into the $\mathrm{Tn} 7$ site of the chromosome. Individual data points for each assay are plotted for each strain, with boxes representing 2 standard deviations and means plotted as horizontal blue lines at the center of the box plots. Motility and colony spreading values are normalized so that the value of DBL1059 is 1. Letters above each box plot indicate that mean values are significantly different at $P<0.01$ according to Tukey's HSD test. Swimming motility (A) and colony spreading (B) phenotypes in an $a m r Z$ deletion strain can be complemented by alleles of amrZ from either $P$. stutzeri or $P$. aeruginosa.

colony spreading defects in P. stutzeri, demonstrating that the two versions of this protein regulate motility in a similar way. Therefore, it does not appear that the phenotypic switch in regulation by AmrZ is due to amino acid changes in the protein itself, which strongly suggests that differential regulation of motility across these strains is mediated by loci downstream of AmrZ regulation. It is also possible that changes within AmrZ-interacting proteins could explain differences in phenotype, but there are currently no proteins known to interact in this way. We also note that, although the initial results of this experiment suggest that the $P$. aeruginosa allele could be hyperactive, we have created independent versions of each of these strains and find that there is no consistent difference in motility between the two alleles of $a m r Z$ during complementation at the Tn7 site (https://figshare.com/s/2a79a1e48fa851d51577).

Interestingly, we observed that a mutant allele of $a m r Z$ (AmrZV21L) arose and swept to fixation during an ongoing evolutionary passage experiment using $P$. stutzeri and carried out within our lab (data not shown; see also Fig. 4C). This mutation occurs within a region of AmrZ known to be involved in dimerization in P. aeruginosa (8) and therefore possibly affects interactions between independent copies of this protein in P. stutzeri. Surprisingly, we demonstrate here that the mutant AmrZV21L allele is able to complement the colony spreading phenotype but not the swimming motility phenotype within our amrZ-knockout strains (Fig. 3). This result suggests that, even though colony spreading and swimming motility are both positively regulated by AmrZ within $P$. stutzeri, the mechanisms of positive regulation for these two phenotypes are at least partially independent. We do not yet know how regulation of these pathways mechanistically differs, but the position of the mutation suggests that dimerization is required only for positive regulation of swimming motility. Recent demonstrations have shown that AmrZ can indirectly alter pools of cyclic di-GMP $(7,16)$, and since cyclic di-GMP is a critical signaling molecule for some types of motility across pseudomonads (20), it is also possible that regulatory independence of these phenotypes reflects differential influence of cyclic di-GMP. At the very least, that these two phenotypes are independently regulated by $A m r Z$ speaks to the evolutionary flexibility of positive regulation by AmrZ. 
A)

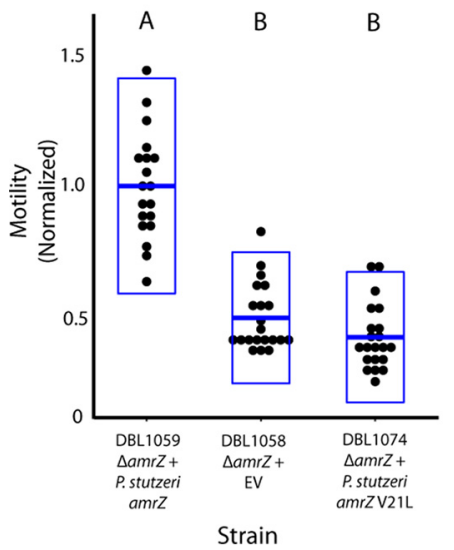

B)

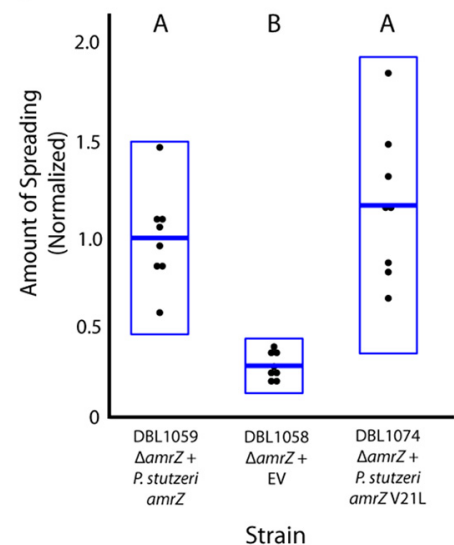

FIG 3 AmrZ independently regulates swimming motility and colony spreading. Strains DBL1058, DBL1059, and DBL1074 are derived from strain DBL830 ( $\triangle a m r Z)$. DBL1058 contains an empty-vector gentamicin resistance cassette, while DBL1059 contains amrZ (native promoter) integrated into the $\mathrm{Tn} 7$ site of the chromosome. DBL1074 contains a mutant version of amrZ (AmrZV21L) integrated into the Tn7 site of the chromosome. Individual data points for each assay are plotted for each strain, with boxes representing 2 standard deviations and means plotted as horizontal blue lines at the center of the box plots. Motility and colony spreading values are normalized so that the value of DBL1059 is 1. Letters above each box plot indicate that mean values are significantly different at $P<0.01$ according to Tukey's HSD. (A) The wild-type version of AmrZ can complement the swimming motility defect of DBL830, but the AmrZV21L mutant version cannot. (B) Both the wild-type version of AmrZ and the AmrZV21L mutant version can complement a colony spreading deficiency of DBL830.

Given the challenges inherent in defining precise types of bacterial motility, it is possible that the colony spreading observed in this report is phenotypically correlated with twitching or swarming motility. P. stutzeri amrZ deletion mutant colonies appear "rough" and "dry" during growth on King's B (KB) medium compared to wild-type strains but are as competent for natural transformation on saltwater LB medium (suggesting a functional pilus [21]) as wild-type strains (data not shown). It is therefore likely that phenotypic differences in colony spreading in the $a m r Z$ mutants are due to lack of production of molecules like surfactants rather than direct changes in either flagellar or pilus-dependent motility.

To demonstrate possible evolutionary scenarios explaining differential regulation of swimming motility by AmrZ across pseudomonads, we inferred phylogenies by using critical strains for which swimming motility effects of AmrZ have been evaluated as well as an outgroup (Azotobacter vinelandii) that also contains a version of this regulator. As one can see in Fig. 4B, there are three equally parsimonious scenarios for the evolution of positive regulation of swimming motility by AmrZ. Under the first scenario (labeled 1), AmrZ is a negative regulator of swimming motility in the ancestor of pseudomonads, and positive regulation has independently evolved twice. Under the second scenario (labeled 2), the ancestral version of AmrZ is a positive regulator of swimming motility, and negative regulation has independently evolved twice. Under the third scenario, AmrZ is a negative regulator of swimming motility in an ancestral strain, evolves to be a positive regulator before the split of $P$. stutzeri and $P$. syringae, and subsequently evolves as a negative regulator again in $P$. fluorescens. That the phylogeny of AmrZ matches that of RpoD/GyrB, coupled with the ability of the $P$. aeruginosa allele to complement $P$. stutzeri phenotypes, rules out scenarios implicating horizontal gene transfer of $a m r Z$ in changes to the mode of regulation for swimming motility. In any case, this example definitively demonstrates that the mode of regulation for AmrZ for swimming motility has changed at least twice independently.

Only a few bacterial transcriptional regulators are known to act as both activators and repressors of gene expression. One of these proteins, AmrZ, has been canonically considered a negative regulator of motility across pseudomonads with one exception shown to date $(7,15,17)$. We provide evidence for the evolutionary plasticity of AmrZ, 
A) 0.2

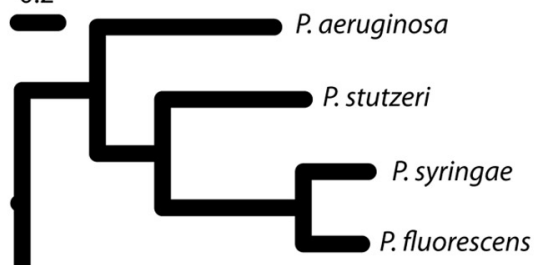

A. vinelandii
B)

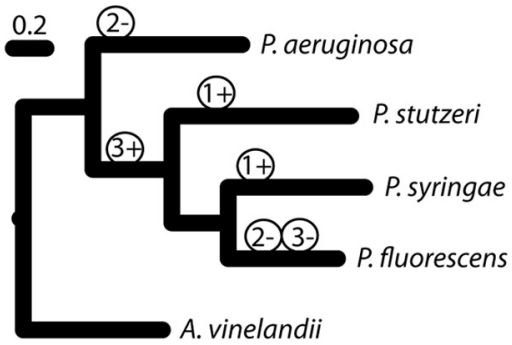

C)

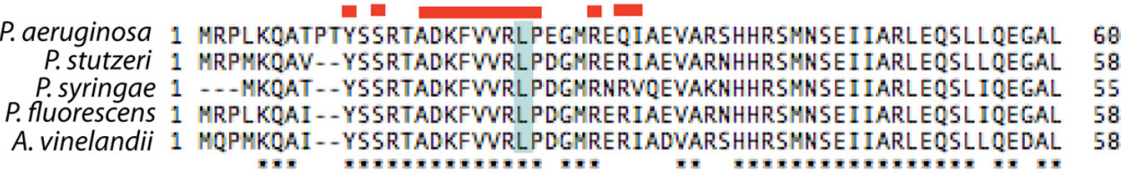

P. aeruginosa 61 QDNLGVRLDSPELSLHERELLQRFRQLTHRQQNALVALIAHDAELAQA - . . . . - 108 P. stutzeri 59 DDDSAMRLDSPELTLHERELLQRFRQLAHRQQNAL ISLIAQDTESAKDED .... 108 P. syringae 56 GDEPSLRLDSPELSLHERELLQRFROLSHROONALVSL IAHDTELASEES - . . - 185 P. fluorescens 59 GEELSMRLDSPELSLHERELLORFROLSHROONALVSLIAHDAEMAADAT - . - 188 A. vinelandii 59 GDDLSLRLDSPELSLHERELLQRFRQLTRRQQNALVALIAQDSELATEGATTEQ 112

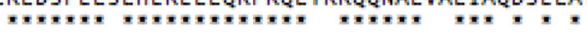

FIG 4 The mode of regulation of $A m r Z$ in swimming motility has shifted at least twice across Pseudomonas. ( $A$ and B) Bayesian phylogenies were built using either RpoD/GyrB (A) or AmrZ (B) for four strains of Pseudomonas for which the role of AmrZ in swimming motility has been evaluated and using Azotobacter vinelandii as an outgroup. Support for all nodes on each phylogeny is $>0.95$ posterior probability, and the phylogeny of RpoD/GyrB matches that built from whole-genome information (data not shown). (B) Three equally parsimonious scenarios, labeled 1 to 3 , for the evolution of mode of regulation of AmrZ for swimming motility are overlaid onto the phylogeny of AmrZ. "+" indicates that positive regulation arose during each of the three scenarios, while "-" indicates that negative regulation arose. Parsimonious inferences follow logically from an assumption that AmrZ is a negative regulator of swimming motility (scenarios 1 and 3 ) or a positive regulator (scenario 2 ) in $A$. vinelandii. Given these starting assumptions, the number of regulatory switches required to explain the phenotypic data given phylogenies was deduced by hand. (C) Protein alignments of AmrZ for the strains used in phylogenetic comparisons are shown. Red lines above this alignment indicate amino acids that have been shown to be involved in dimerization of AmrZ (according to reference 8). We also highlight, in light blue, the amino acid that has changed in the AmrZV21L allele.

by demonstrating that this protein acts independently as a positive regulator of swimming motility and colony spreading phenotypes in P. stutzeri. Therefore, we definitively show that there have been at least two independent shifts in function for AmrZ in the context of swimming motility across pseudomonads. Closer investigation of mechanistic shifts in dual-function regulators like AmrZ could provide unique insights into how transcriptional pathways are rewired between closely related species.

\section{MATERIALS AND METHODS}

Bacterial strains, plasmids, and culture conditions. All strains and plasmids used in the study are listed in Table 1. DBL332 was selected as a rifampin-resistant isolate of strain 23a24 (22). Strain propagation of $P$. stutzeri largely took place at $27^{\circ} \mathrm{C}$ in King's B medium supplemented with rifampin. Antibiotics were used in the following concentrations where appropriate: $50 \mu \mathrm{g} / \mathrm{ml} \mathrm{rifampin,} 10 \mu \mathrm{g} / \mathrm{ml}$ tetracycline, $40 \mu \mathrm{g} / \mathrm{ml}$ nitrofurantoin, $10 \mu \mathrm{g} / \mathrm{ml}$ gentamicin, and $20 \mu \mathrm{g} / \mathrm{ml}$ kanamycin.

Creation of $\boldsymbol{a m r Z}$ mutants. Creation of the construct for deleting $a m r Z$ is described in depth at https://doi.org/10.6084/m9.figshare.3204178.v1. Briefly, regions upstream and downstream of $a m r Z$ in $P$. stutzeri DBL332 were amplified and spliced using overlap PCR. This fragment was recombined into pDONR207 using BP recombinase to create pDBL63. pDBL63 was then recombined with pMTN1907 to create pDBL64, which can be used to cleanly delete gene regions within $P$. stutzeri $(22,23)$. Once pDBL64 was created, it was mated into either DBL332 or DBL390 through triparental mating with the helper strain containing plasmid pRK2013 (DAB42). DBL390 is a gentamicin-resistant version of DBL332 where lacZ has been integrated into the Tn7 site using pUC18-mini-TnTT-Gm-LacZ prior to conjugation with PDBL64 and therefore represents independent deletion of $a m r Z$. After mating, tetracycline-resistant recombinants in both DBL332 and DBL390 backgrounds were selected on LB medium. Each recombinant was grown overnight in LB medium supplemented with rifampin and spread on KB plates containing $5 \%$ sucrose. Isolates of $P$. stutzeri DBL332 and DBL390 in which $a m r Z$ has been deleted are distinguishable when grown on KB medium because they manifest as rough colonies. Clean deletion of $a m r Z$ was confirmed through PCR with primers DBL383 and DBL384. 
TABLE 1 Strains and plasmids

\begin{tabular}{|c|c|c|}
\hline Strain or plasmid number & Strain or plasmid description & Citation \\
\hline \multicolumn{3}{|l|}{ Strains } \\
\hline DBL390 & P. stutzeri DBL390, Rifr Gent ${ }^{r}$ LacZ $^{+}$ & 22 \\
\hline DBL831 & P. stutzeri DBL390 with pDBL64 integrated, Rifr ${ }^{\text {Gent }}{ }^{r}$ LacZ $^{+}$Tet $^{r}$ Suc ${ }^{s}$ & This work \\
\hline DBL1052 & P. stutzeri DBL332 with deletion of $a m r Z$, Rifr & This work \\
\hline DBL1053 & P. stutzeri DBL390 with deletion of $a m r Z$, Rifr $^{\text {Gentr }}$ LacZ $^{+}$ & This work \\
\hline DBL1060, DBL1252 & P. stutzeri DBL1052 with Tn7 transposition from pDBL94, Rifr Gentr ${ }^{r}$ & This work \\
\hline DBL1074 & P. stutzeri DBL1052 with Tn7 transposition from pDBL95, AmrZL21V, Rifr Gent ${ }^{r}$ & This work \\
\hline DBL1118 & P. stutzeri DBL332 with pDBL99 integrated, Rifr Tetr $^{r}$ Suc $^{s}$ & This work \\
\hline DBL1168 & P. stutzeri DBL332 with deletion of flgK, Rifr & This work \\
\hline \multicolumn{3}{|l|}{ Plasmids } \\
\hline pME3280a & Tn7 empty vector & 28 \\
\hline pDBL92 & $P$. aeruginosa amrZ ORF with stop codon and $P$. stutzeri promoter in pDONR207 & This work \\
\hline pDBL93 & P. stutzeri amrZ ORF with stop codon and P. stutzeri promoter in Tn7-GW & This work \\
\hline pDBL94 & P. aeruginosa amrZ ORF with stop codon and $P$. stutzeri promoter in Tn7-GW & This work \\
\hline pDBL95 & P. stutzeri amrZC61G ORF with stop codon and $P$. stutzeri promoter in pDONR207 & This work \\
\hline pDBL96 & P. stutzeri amrZC61G ORF with stop codon and P. stutzeri promoter in Tn7-GW & This work \\
\hline pDBL99 & P. stutzeri flgK deletion construct in pDONR207 & This work \\
\hline pDBL100 & P. stutzeri flgK deletion construct in pMTN1907 & This work \\
\hline pMTN1907 & Gateway destination vector for generating deletions in $P$. stutzeri & 23 \\
\hline
\end{tabular}

Complementation of $\mathbf{a m r Z}$. Creation of complementation constructs is described at https://doi.org/ 10.6084/m9.figshare.3365263.v1. Briefly, the $a m r Z$ open reading frame (ORF) (including the stop codon) and native promoter for $a m r Z$ were amplified from strain DBL332. This fragment was purified and recombined into pDONR207 using BP Clonase to create PDBL91. This construct was then recombined from pDBL91 into a Tn7 transposon on the Gateway destination vector pTn7-GW (J. Chang, unpublished data) to create PDBL93. Last, this construct was transposed onto the DBL1052 chromosome (a clean deletion of $a m r Z$ in the DBL332 background) through natural transformation after mixing cells with both pDBL93 and pTNS2. The complementation construct for the mutant allele of $a m r Z$ (DBL1074, AmrZV21L) was created using these same primer sets by amplifying this region from a strain naturally containing the allele and using BP Clonase to create plasmid PDBL95. The resulting destination vector from this construct (pDBL96) was used for transposition in the same way as the wild-type version described above. A complementation construct using the $P$. aeruginosa amrZ ORF was created in a similar way except that the BP reaction was carried out with a synthesized gBlock (Integrated DNA Technologies, Inc., Coralville, IA) to create pDBL92. Expression of the $P$. aeruginosa allele of $a m r Z$ in this construct is driven by the same promoter sequence (from $P$. stutzeri) as in PDBL93. pDBL94 was created through an LR reaction involving pDBL92 and pTn7-GW and transformed into DBL1052.

Creation of $f \mathbf{g} K$ mutant strain. In order to test whether a knockout of $a m r Z$ was partially motile or completely immotile, we constructed a strain of $P$. stutzeri (DBL1157) in which flgK had been completely deleted from the genome. A file describing the creation of the flgK mutant can be found at https:// figshare.com/s/a9b633c3dcb7acbadcfe.

Motility assays. Following overnight growth in LB medium and two washes with $1 \mathrm{ml} 10 \mathrm{mM} \mathrm{MgCl}$, the optical density at $600 \mathrm{~nm}\left(\mathrm{OD}_{600}\right)$ of each strain was standardized at 1.0 in $10 \mathrm{mM} \mathrm{MgCl}$. A blunt-ended toothpick was dipped into this inoculum and then dipped into the center of a 12-well tissue culture plate containing one-half-strength LB medium with $0.25 \%$ agar. Plates were covered with Parafilm and incubated for either 24 or $48 \mathrm{~h}$ at room temperature (indicated in figure legends), after which they were scanned at 600 dots per inch (dpi). Images were imported into ImageJ, and the pixel area of each strain was quantified. Data for all assays can be found at https://figshare.com/s/ $\mathrm{bd} 8 \mathrm{~d} 3 \mathrm{~d} 0 \mathrm{a} 12 \mathrm{~b} 8 \mathrm{c} 9 \mathrm{f} 36 \mathrm{~b} 5 \mathrm{~b}$. The motility of each strain for each assay was normalized to a control strain within the same experiment, with figure legends identifying the normalized strain, so that motilities across experiments were comparable. Normalization did not affect overall statistical outcomes (data not shown). Each set of experiments was independently run twice, with at least 8 replicates per experiment. Statistical tests were carried out in R (24), with one-way analyses of variance (ANOVAs) with "normalized motility" as the dependent variable and "strain" as a fixed-effect independent variable. Inclusion of "assay" as a second independent variable did not affect overall statistical outcomes (data not shown). Tukey's honestly significant difference (HSD) test was then used to classify strain effects.

Colony spreading assays. Following overnight growth in KB medium and two washes with $1 \mathrm{ml}$ $10 \mathrm{mM} \mathrm{MgCl}_{2}$, the $\mathrm{OD}_{600}$ of each strain was standardized at 1.0 in $10 \mathrm{mM} \mathrm{MgCl}_{2}$. Ten microliters of this 
suspension was pipetted onto King's B (KB) medium with 1.5\% agar. Each experimental plate contained all strains within a given comparison (Fig. 1C). Plates were covered in Parafilm and incubated for $72 \mathrm{~h}$ at room temperature. At three time points (after 24, 48, and $72 \mathrm{~h}$ of growth), plates were scanned at 600 dpi. Images were imported into ImageJ, and the pixel area of each strain was quantified. Data for all assays can be found at https://figshare.com/s/bd8d3d0a12b8c9f36b5b. The amount of spreading was calculated by taking the difference in area between 24 and 72 hours for each strain on each plate, except for one case where spreading was calculated between 48 and 72 hours. Spreading of each strain for each assay was normalized to a strain from the same plate within the same experiment, with figure legends identifying the normalized strain, so that motilities across experiments were comparable. Normalization did not affect overall statistical outcomes (data not shown). Each set of experiments was independently run twice, with at least 2 (but usually 4) replicated plates per experiment. Statistical tests were carried out in R (24), with one-way ANOVAs with "normalized spreading" as the dependent variable and "strain" as a fixed-effect independent variable. Inclusion of "assay" as a second independent variable did not affect overall statistical outcomes (data not shown). Tukey's HSD test was then used to classify strain effects.

Phylogenetic comparisons. Bayesian phylogenies were created using protein sequences from conserved genes from each strain, obtained from the JGI Integrated Microbial Genomes (IMG) database (25). Strains used for this comparison were P. stutzeri $23 a 24$ (IMG identifier [ID] 2565956579), P. fluorescens F113 (IMG ID 2511231156), P. syringae pv. tomato DC3000 (IMG ID 2508501074), P. aeruginosa PAO1 (IMG ID 637000218), and Azotobacter vinelandii CA (IMG ID 2541047084).

Protein sequences for GyrB and RpoD were used to infer phylogeny of "housekeeping" genes. GyrB and RpoD sequences were independently aligned using Clustal X (26) and then concatenated. MrBayes was used for Bayesian phylogenetic analysis on these sequences (27), using flat priors and a burn-in period of 25,000 generations. In each case, convergence of the run occurred before 100,000 total generations. Phylogenies built using whole-genome sequences for these strains completely agree with the reported trees (data not shown). A phylogeny for AmrZ was built in the same way as that of RpoD/GyrB. Alignments and output files from MrBayes can be found on Figshare at https://figshare.com/ $\mathrm{s} / \mathrm{bd} 8 \mathrm{~d} 3 \mathrm{~d} 0 \mathrm{a} 12 \mathrm{~b} 8 \mathrm{c} 9 \mathrm{f} 36 \mathrm{~b} 5 \mathrm{~b}$.

Data availability. Data underlying all statistical analyses, sequence alignments, and evolutionary analyses within this paper are available through Figshare at https://doi.org/10.6084/m9.figshare.3365299 .v4 and https://doi.org/10.6084/m9.figshare.5965417.v1.

\section{ACKNOWLEDGMENTS}

We thank Brian Smith and Kevin Hockett for technical assistance.

This research was funded through startup funds to D.A.B. from the University of Arizona, as well as a SEED grant from the University of Arizona Foundation.

\section{REFERENCES}

1. Dekel E, Alon U. 2005. Optimality and evolutionary tuning of the expression level of a protein. Nature 436:588-592. https://doi.org/10.1038/ nature03842.

2. Stoebel DM, Dean AM, Dykhuizen DE. 2008. The cost of expression of Escherichia coli lac operon proteins is in the process, not in the products. Genetics 178:1653-1660. https://doi.org/10.1534/genetics.107.085399.

3. Price MN, Wetmore KM, Deutschbauer AM, Arkin AP. 2016. A comparison of the costs and benefits of bacterial gene expression. PLoS ONE 11: e0164314. https://doi.org/10.1371/journal.pone.0164314.

4. Perez JC, Groisman EA. 2009. Evolution of transcriptional regulatory circuits in bacteria. Cell 138:233-244. https://doi.org/10.1016/j.cell.2009 .07 .002 .

5. Mitchell A, Romano GH, Groisman B, Yona A, Dekel E, Kupiec M, Dahan O, Pilpel Y. 2009. Adaptive prediction of environmental changes by microorganisms. Nature 460:220-224. https://doi.org/10.1038/nature08112.

6. McAdams HH, Srinivasan B, Arkin AP. 2004. The evolution of genetic regulatory systems in bacteria. Nat Rev Genet 5:169-178. https://doi .org/10.1038/nrg1292.

7. Jones CJ, Newsom D, Kelly B, Irie $Y$, Jennings LK, Xu B, Limoli DH, Harrison JJ, Parsek MR, White P, Wozniak DJ. 2014. ChIP-Seq and RNASeq reveal an AmrZ-mediated mechanism for cyclic di-GMP synthesis and biofilm development by Pseudomonas aeruginosa. PLoS Pathog 10:e1003984. https://doi.org/10.1371/journal.ppat.1003984.

8. Pryor EE, Waligora EA, Xu B, Dellos-Nolan S, Wozniak DJ, Hollis T. 2012. The transcription factor AmrZ utilizes multiple DNA binding modes to recognize activator and repressor sequences of Pseudomonas aeruginosa virulence genes. PLoS Pathog 8:e1002648. https://doi.org/10.1371/journal .ppat.1002648.

9. Xu B, Ju Y, Soukup RJ, Ramsey DM, Fishel R, Wysocki VH, Wozniak DJ. 2016. The Pseudomonas aeruginosa AmrZ C-terminal domain mediates tetramerization and is required for its activator and repressor functions. Environ Microbiol Rep 8:85-90. https://doi.org/10.1111/1758-2229.12354.
10. Tart AH, Blanks MJ, Wozniak DJ. 2006. The AlgT-dependent transcriptional regulator AmrZ (AlgZ) inhibits flagellum biosynthesis in mucoid, nonmotile Pseudomonas aeruginosa cystic fibrosis isolates. J Bacteriol 188:6483-6489. https://doi.org/10.1128/JB.00636-06

11. Falcone M, Ferrara S, Rossi E, Johansen HK, Molin S, Bertoni G. 2018. The small RNA ersA of Pseudomonas aeruginosa contributes to biofilm development and motility through post-transcriptional modulation of AmrZ. Front Microbiol 9:238. https://doi.org/10.3389/fmicb.2018.00238.

12. Allsopp LP, Wood TE, Howard SA, Maggiorelli F, Nolan LM, Wettstadt S, Filloux A. 2017. RsmA and AmrZ orchestrate the assembly of all three type VI secretion systems in Pseudomonas aeruginosa. Proc Natl Acad Sci U S A 114:7707-7712. https://doi.org/10.1073/pnas.1700286114.

13. Baynham PJ, Brown AL, Hall LL, Wozniak DJ. 1999. Pseudomonas aeruginosa AlgZ, a ribbon-helix-helix DNA-binding protein, is essential for alginate synthesis and $\operatorname{alg} D$ transcriptional activation. Mol Microbiol 33:1069-1080. https://doi.org/10.1046/j.1365-2958.1999.01550.x.

14. Baynham PJ, Ramsey DM, Gvozdyev BV, Cordonnier EM, Wozniak DJ. 2006. The Pseudomonas aeruginosa ribbon-helix-helix DNA-binding protein AlgZ (AmrZ) controls twitching motility and biogenesis of type IV pili. J Bacteriol 188:132-140. https://doi.org/10.1128/JB.188.1.132-140 .2006 .

15. Martínez-Granero FM, Redondo-Nieto M, Vesga P, Martín M, Rivilla R. 2014. AmrZ is a global transcriptional regulator implicated in iron uptake and environmental adaption in $P$. fluorescens F113. BMC Genomics 15:237. https://doi.org/10.1186/1471-2164-15-237.

16. Muriel C, Arrebola E, Redondo-Nieto M, Martínez-Granero F, Jalvo $B$, Pfeilmeier S, Blanco-Romero E, Baena I, Malone JG, Rivilla R, Martín M. 2018. AmrZ is a major determinant of c-di-GMP levels in Pseudomonas fluorescens F113. Sci Rep 8:1979. https://doi.org/10.1038/s41598-018 -20419-9.

17. Prada-Ramírez HA, Pérez-Mendoza D, Felipe A, Martínez-Granero F, Rivilla R, Sanjuán J, Gallegos MT. 2016. AmrZ regulates cellulose production 
in Pseudomonas syringae pv. tomato DC3000. Mol Microbiol 99:960-977. https://doi.org/10.1111/mmi.13278.

18. Lalucat J, Bennasar A, Bosch R, García-Valdés E, Palleroni NJ. 2006. Biology of Pseudomonas stutzeri. Microbiol Mol Biol Rev 70:510-547. https://doi.org/10.1128/MMBR.00047-05.

19. Gomila M, Peña A, Mulet M, Lalucat J, García-Valdés E. 2015. Phylogenomics and systematics in Pseudomonas. Front Microbiol 6:214. https:// doi.org/10.3389/fmicb.2015.00214.

20. Caiazza NC, Merritt JH, Brothers KM, O'Toole GA. 2007. Inverse regulation of biofilm formation and swarming motility by Pseudomonas aeruginosa PA14. J Bacteriol 189:3603-3612. https://doi.org/10.1128/JB.01685-06.

21. Graupner S, Frey V, Hashemi R, Lorenz MG, Brandes G, Wackernagel W. 2000. Type IV pilus genes pilA and pilC are required for genetic transformation, and pilA can be replaced by corresponding genes from nontransformable species. J Bacteriol 182:2184-2190. https://doi.org/10 $.1128 / J B .182 .8 .2184-2190.2000$

22. Romanchuk A, Jones CD, Karkare K, Moore A, Smith BA, Jones C, Dougherty K, Baltrus DA. 2014. Bigger is not always better: transmission and fitness burden of $1 \mathrm{MB}$ Pseudomonas syringae megaplasmid pMPPla107. Plasmid 73:16-25. https://doi.org/10.1016/j.plasmid.2014.04.002.

23. Baltrus DA, Nishimura MT, Dougherty KM, Biswas S, Mukhtar MS, Vicente J, Holub EB, Dangl JL. 2012. The molecular basis of host specialization in bean pathovars of Pseudomonas syringae. Mol Plant Microbe Interact 25:877-888. https://doi.org/10.1094/MPMI-08-11-0218.
24. R Core Team. 2013. R: a language and environment for statistical computing. R Foundation for Statistical Computing, Vienna, Austria. http:// www.R-project.org.

25. Markowitz VM, Chen IM, Palaniappan K, Chu K, Szeto E, Pillay M, Ratner A, Huang J, Woyke T, Huntemann M, Anderson I, Billis K, Varghese N, Mavromatis K, Pati A, Ivanova NN, Kyrpides NC. 2014. IMG 4 version of the integrated microbial genomes comparative analysis system. Nucleic Acids Res 42:D560-D567. https://doi.org/10.1093/nar/gkt963.

26. Larkin MA, Blackshields G, Brown NP, Chenna R, McGettigan PA, McWilliam H, Valentin F, Wallace IM, Wilm A, Lopez R, Thompson JD, Gibson TJ, Higgins DG. 2007. Clustal W and Clustal X version 2.0. Bioinformatics 23:2947-2948. https://doi.org/10.1093/bioinformatics/btm404.

27. Ronquist $F$, Teslenko $M$, van der Mark $P$, Ayres DL, Darling A, Höhna S, Larget B, Liu L, Suchard MA, Huelsenbeck JP. 2012. MrBayes 3.2: efficient Bayesian phylogenetic inference and model choice across a large model space. Syst Biol 61:539-542. https://doi.org/10.1093/sysbio/ sys029.

28. Schnider-Keel U, Seematter A, Maurhofer M, Blumer C, Duffy B, GigotBonnefoy C, Reimmann C, Notz R, Défago G, Haas D, Keel C. 2000. Autoinduction of 2,4-diacetylphloroglucinol biosynthesis in the biocontrol agent Pseudomonas fluorescens $\mathrm{CHAO}$ and repression by the bacterial metabolites salicylate and pyoluteorin. J Bacteriol 182:1215-1225. https://doi.org/10.1128/JB.182.5.1215-1225.2000. 